\title{
Deciphering nifedipine in vivo delivery from modified release dosage forms: Identification of food effect
}

\author{
MARIJA ILIĆ* \\ IVAN KOVAČEVIĆ \\ JELENA PAROJČIĆ \\ Department of Pharmaceutical \\ Technology, University of Belgrade \\ Faculty of Pharmacy, Serbia
}

Accepted September 2, 2015

\begin{abstract}
With the increased reliance on in vitro dissolution testing as an indicator of in vivo drug behavior and the trend towards the in silico modeling of dosage form performance, the need for bioperformance dissolution methodology development has been enhanced. Determination of the in vivo drug delivery profile is essential for the bioperformance dissolution test development and in vitro/in vivo correlation modeling, as well as the understanding of absorption mechanisms. The aim of this study was to compare different methods in terms of their usefulness and applicability in deciphering in vivo delivery of nifedipine administered in modified release dosage forms. A detailed survey of publications on nifedipine pharmacokinetics was done and used to identify the magnitude of food effect. In vitro dissolution testing was performed under various experimental conditions. Obtained results indicate the potential for using the developed in silico model coupled with discriminative in vitro dissolution data for identification of the in vivo drug product behavior.
\end{abstract}

Keywords: absorption, dissolution, food effect, in silico modeling, deconvolution, GastroPlus ${ }^{\mathrm{TM}}$

Drug absorption from the gastrointestinal tract is a complex process affected by a number of physicochemical, pharmaceutical and physiological factors related to the drug substance properties, dosage form characteristics, as well as the conditions encountered in vivo after drug administration. Meal and drug co-administration have been recognized as an additional factor contributing to this complexity, since the presence of food within the gastrointestinal tract can markedly alter oral drug bioavailability, changing the rate and/ or extent of drug absorption, presystemic metabolism and systemic drug clearance $(1,2)$. The need for fed state bioequivalence studies for both the modified release (MR) as well as immediate release (IR) dosage forms has been recognized by regulatory authorities (3-5). Identification of the time course of drug delivery in vivo is essential to understand the absorption mechanisms and potential interactions encountered, design of dosage forms with

\footnotetext{
*Correspondence; e-mail: marija@pharmacy.bg.ac.rs
} 
the targeted product profile and in vitro/in vivo correlation (IVIVC) modeling. In vivo drug delivery after oral administration is difficult to measure. Therefore, different approaches have been developed with the aim to reconstruct the time course of in vivo drug delivery based on the available pharmacokinetic data (i.e., plasma concentration-time profile). Conventional pharmacokinetic analysis based on the mass balance approaches and compartmental analysis, i.e., Wagner-Nelson and Loo-Riegelman methods, have been most frequently used, as well as model-independent approaches using numerical deconvolution. The advanced knowledge gained about the interplay of different factors influencing drug absorption has fostered the development of predictive models for oral drug absorption such as physiologically based pharmacokinetic models $(6,7)$ and physiologically relevant in vitro dissolution methods (8). Integration of in vitro and in silico approaches is expected to accelerate drug development and improve clinical product performance $(7,8)$. Depending on the method of analysis employed, different in vivo drug delivery profiles (iDDP) may result. This may have further implications for the selection of bioperformance dissolution methodology and IVIVC model development.

Regulatory approach to the in vitro characterization of modified release dosage forms has been recently updated in revised guidances to industry $(5,9)$. It is recommended that " an in vitro dissolution test is developed which is able to detect changes which may have an effect on the efficacy or safety of the product « and that "a link from pharmacokinetic parameters through in vivo drug release to in vitro dissolution rate should be established « (9). Use of different dissolution conditions is anticipated in order to determine the sensitivity of a formulation to the expected physiological environment after administration. Various conditions regarding medium composition and $\mathrm{pH}$, different apparatus and agitation intensities should be employed. Concerns about dose dumping led to the regulatory recommendation for in vitro investigation of alcohol-induced dose dumping, i.e., »in vitro drug release in alcohol solution « (5). It is also anticipated that »the use of biorelevant media may improve the correlation to in vivo data and may detect a potential food effect« (9). The potential of viscous media to reflect food effect has also been investigated $(10,11)$.

Nifedipine is a poorly soluble drug exhibiting a $\mathrm{pH}$ independent solubility profile and high permeability through the gastrointestinal tract $(12,13)$, meeting the criteria for a Class 2 compound according to the Biopharmaceutics Classification System (BCS). Numerous nifedipine modified release formulations have been marketed in order to improve therapeutic compliance in chronic treatment of hypertension and angina pectoris and to reduce the concentration-related adverse effects associated with peak plasma levels $(12,14)$. Literature data on nifedipine pharmacokinetics after drug administration with or without food are available from a number of sources. It has been reported that nifedipine food effect may be formulation specific (14-17). Such data imply that the drug/food interactions observed are physicochemically based and emphasize the importance of bioperformance in vitro testing for the prediction of in vivo behavior of a drug product $(10,14)$.

The aim of the present study was to compare different deconvolution methods (LooRiegelman method, numerical deconvolution and in silico gastrointestinal simulation) in terms of their usefulness and applicability in deciphering in vivo delivery of nifedipine administered in modified release dosage forms. A detailed survey of the literature data on nifedipine pharmacokinetics in fed and fasted states was done and the available data was used to estimate the in vivo drug delivery profile and identify the magnitude of food effect. In vitro dissolution testing of commercially available osmotic controlled-release tablets and 
hydrophilic matrix tablets was conducted under various experimental conditions in order to assess the effect of dissolution media on nifedipine release from different modified release dosage forms and its further use as input function for in silico modeling.

\section{EXPERIMENTAL}

\section{In vivo data}

Deciphering the time course of nifedipine in vivo delivery by applying various deconvolution methods was performed using the same set of in vivo data taken from the literature. Data on nifedipine pharmacokinetics following administration of osmotic controlled-release tablets under fasted and fed state conditions reported in the study of Schug et al. (14) were evaluated as the response function. Plasma concentration-time $(c-t)$ profiles following intravenous and/or oral administration of a nifedipine immediate-release (IR) capsule reported by Rashid et al. (18) were used as the reference/weighting function. Furthermore, a detailed literature survey on the published data about nifedipine pharmacokinetics and food effect was conducted and the data collected were employed for the in vivo drug delivery analysis.

\section{Pharmacokinetic analysis}

Conventional pharmacokinetic analyses, i.e., Loo-Riegelman and numerical deconvolution, were performed using the Kinetica 5.0 software (Thermo Fisher Scientific). The Loo-Riegelman method may be used to quantify the absorption kinetics when the corresponding intravenous data suggest bi-exponential decay in the distribution and elimination profile (19). This method requires data following both oral and intravenous administration of the drug to the same subject. The percent drug absorbed can be calculated by the following general equation based on the Loo-Riegelman method:

$$
F a(t)=\frac{C+k_{10} \int_{0}^{t} C d t+k_{12} \cdot e^{-k_{21} \cdot t} \int_{0}^{t} C \cdot e^{k_{21} \cdot t} d t}{k_{10} \int_{0}^{\infty} C d t}
$$

Numerical deconvolution is a model-independent method for determining absorption rates based on the superposition and time invariance principles. In the convolution integral (Eq. 2), the response function corresponds to the sum of responses related to the discrete unit impulses shifted over time. As an inverse function, deconvolution enables calculation of the input function provided that the response function and weighting function (which may be described as the response to the unit impulse, such as the i.v. bolus injection) are available.

$$
C(t)=\int_{0}^{t} f(\tau) \cdot C_{\delta}(t-\tau) d \tau
$$


where $C(t)$ is the response function; $C_{\delta}$ is the response to a unit impulse (weighting function) and $f$ is the input function.

This method requires no assumptions regarding the number of compartments in the model or absorption kinetics; however, linear distribution and elimination kinetics are assumed. Similarly to the Loo-Riegelman method, numerical deconvolution requires data obtained after both oral and intravenous administration in the same subject and assumes no differences in the pharmacokinetics of drug distribution and elimination. Although the i.v. reference is preferred, in the case of modified release preparations, the use of oral solution or immediate release dosage form as a reference would result in the input reflecting the in vivo drug release from the dosage form, which would be advantageous for IVIVC modeling.

For numerical deconvolution, plasma concentration-time profiles reported following administration of nifedipine modified release tablets were used as the response function, while profiles reported following intravenous (i.v.) and/or oral immediate release nifedipine capsule administration (p.o.) were employed as the weighting function. Input function (i.e., in vivo drug delivery profile) resulting from numerical deconvolution based on the i.v. reference as weighting function refers to the drug absorption profile. Numerical deconvolution based on oral reference as the weighting function provides an estimate of the in vivo drug dissolution profile. To take into account unequal sampling intervals of the evaluated plasma profiles, the »Interpolation « option in Kinetica 5.0 Deconvolution analysis mode was selected. When oral reference was used, the default Method option in Kinetica 5.0 Deconvolution analysis 'Extrapolated $\operatorname{Civ}(t=0)$ ' (referring to the i.v. reference) was changed to the Method option »Civ $(t=0)=$ User define value and 'user define value' set as $C=0$ at $t=0$.

\section{Gastrointestinal simulation}

Mechanistic gastrointestinal absorption simulation based on the Advanced Compartmental Absorption and Transit (ACAT) model was performed using the commercially available software GastroPlus ${ }^{\mathrm{TM}}$ version 8.0 (Simulations Plus, Lancaster, California, USA). This model is based on the assumption that the drug in vivo delivery profile may be assessed by combining the drug physicochemical and biopharmaceutical properties with the physiological parameters. It can include all the major processes relevant to drug absorption and elimination, such as nonlinear dose-dependent absorption, first pass metabolism in gut and liver, presence of a limited absorption window for the compounds whose absorption is dependent on influx transporters with narrow regional expression in gut $(7,20)$.

Gastrointestinal absorption simulation was performed in the GastroPlus Single Simulation Mode with the default Absorption Scale Factor (ASF) values in the Physiology section. In order to take into account the potential food effect, drug absorption was simulated using the 'Human-Physiological-Fed' mode in the GastroPlus settings. The gastrointestinal simulation (GIS) model development and validation were described previously (21).

\section{In vitro study}

In vitro drug release studies of commercially available nifedipine osmotic controlledrelease tablets (Adalat OROS, Bayer, Germany) and hydrophilic matrix tablets (Nifelat retard, Zdravlje, Serbia) were conducted in a rotating paddle apparatus (Erweka DT 600, Ger- 
many), protected from light. The dissolution conditions comprised a $500 \mathrm{~mL}$ medium volume and paddle revolution speed of $100 \mathrm{rpm}$ at $37 \pm 0.5^{\circ} \mathrm{C}$. To investigate the influence of different experimental conditions on drug release from selected nifedipine MR products, various dissolution media were employed: (i) physiologically based fasted and fed state gastrointestinal fluids, (ii) viscous media containing $0.5 \%$ hydroxypropylmethylcellulose (HPMC) with $\mathrm{pH}$ 6.8, (iii) alcoholic media presented as $40 \%$ ethanol solution, (iv) purified water and $(v) 1 \%$ aqueous solution of sodium lauryl sulfate (SLS) with $\mathrm{pH}$ 7.5. Physiologically based dissolution media simulating pre- and postprandial states in the stomach and small intestine, namely Fasted State Simulated Gastric Fluid (FaSSGF), Fed State Simulated Gastric Fluid (FeSSGF), Fasted State Simulated Intestinal Fluid (FaSSIF) and Fed State Simulated Intestinal Fluid (FeSSIF) were prepared as described by Jantratid et al. (22). Samples were assayed for nifedipine UV spectrophotometrically or using high-performance liquid chromatography, as described previously (21). For FeSSGF with milk as a major component, $2 \mathrm{~mL}$ of isopropyl alcohol was added to a 3-mL sample, mixed well and the mixture was centrifuged at $3500 \mathrm{rpm}$ for $5 \mathrm{~min}$. The supernatant was used for the assay.

\section{RESULTS AND DISCUSSION}

In vivo drug delivery profiles

Nifedipine in vivo delivery profiles of osmotic controlled-release tablets estimated by applying different methods to the same set of in vivo data are given in Fig. 1.

All the methods employed indicated a certain lag time (up to 3 hours post dose) regardless of food intake. Irrespective of the different underlying assumptions relating to

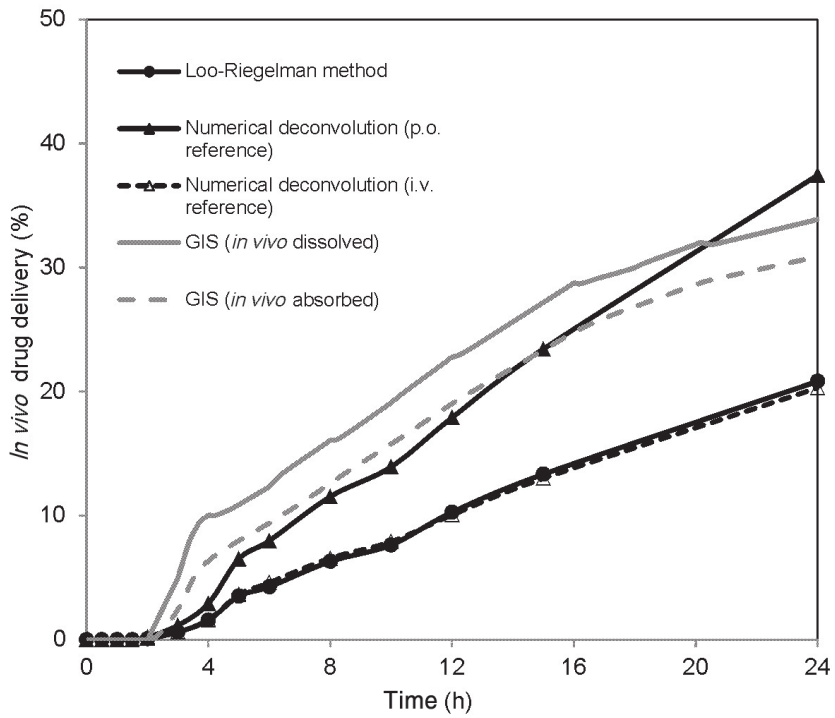

Fig. 1. Nifedipine in vivo delivery profiles of osmotic controlled-release tablets under fasting conditions estimated by different methods. 
each method, the profiles obtained using the Loo-Riegelman method and numerical deconvolution based on the i.v. reference were superimposable, leading to $20 \%$ of the dose dissolved 24 hours after administration. The total amount of nifedipine dissolved in vivo obtained by numerical deconvolution using the nifedipine IR capsule plasma concentration as a reference/weighting function was $50 \%$ after 48 hours post administration. Lower estimates of the in vivo drug delivery, calculated based on the intravenous reference (i.e., the amount of drug available in the systemic circulation), compared to that obtained with the oral capsule plasma profile used as a reference (i.e., the amount of drug dissolved in vivo) reflect the extent of nifedipine "presystemic metabolism» (23). The estimated extent of nifedipine absorption is in accord with the literature data on nifedipine bioavailability reported in the range $45-56 \%$ owing to the first pass effect (23). Nifedipine absorption/ dissolution profiles obtained by gastrointestinal simulation (GIS) were close to the in vivo profile calculated by numerical deconvolution based on the IR reference. Differences between absorption and dissolution profiles estimated by GIS could be related to the nifedipine precipitation in vivo observed for doses above $10 \mathrm{mg}$, as reported by Wagner et al. (13). Although generally recommended as the weighting function in convolution/deconvolution calculations aimed at the development of an IVIVC model, the intravenous reference might not be adequate when deciphering input kinetics of drugs exhibiting extensive presystemic metabolism. Drug delivery profile estimated using the plasma profile observed in vivo after the administration of IR capsule as a reference is indicative of the in vivo drug dissolution. Hence, it should be more advantageous as the targeted profile in the course of IVIVC development and design of the bioperformance dissolution methodology. It was also found that using the input physicochemical and pharmacokinetic parameters in the developed in silico model, it was possible to generate nifedipine absorption and dissolution profiles in vivo, in both fasted and fed state, important for guiding the rationale design or changes of the investigated dosage form.

\section{The extent of food effect}

An overview of the available literature data on pharmacokinetics of a range of nifedipine dosage forms administered in fasted and fed states is given in Table I.

Ratios of the reported pharmacokinetic parameter values in fed vs. fasted state, for a range of nifedipine dosage forms, indicate that drug absorption in the presence of food is formulation specific. Both positive and negative effects of a co-administered meal on the rate and extent of nifedipine absorption have been reported and a number of potential explanations have been suggested. Osmotic controlled-release tablets (studies IVa and VIIa) were designed so as to be robust against the change in the environmental conditions encountered in vivo, which is evident from the ratios of the reported pharmacokinetic parameter values in fed $v s$. fasted state being close to one. The majority of modified release products exhibited a positive food effect in which both $A U C$ and $C_{\max }$ values increased when co-administered with food (studies III, IVb, V, VI, VIIb and VIII). Significantly increased $C_{\max }$ values accompanied with decreased $t_{\max }$ indicated rapid and uncontrolled nifedipine release in the presence of food (i.e., dose dumping) (studies V, VI, VIIb and VIII). A negative food effect was observed in the case of IR capsule formulation, where delayed and incomplete drug absorption was observed when co-administered with food (study I).

Nifedipine pharmacokinetic profiles observed in the fasted and fed state studies and summarized in Table I were further employed in order to calculate the relevant in vivo 


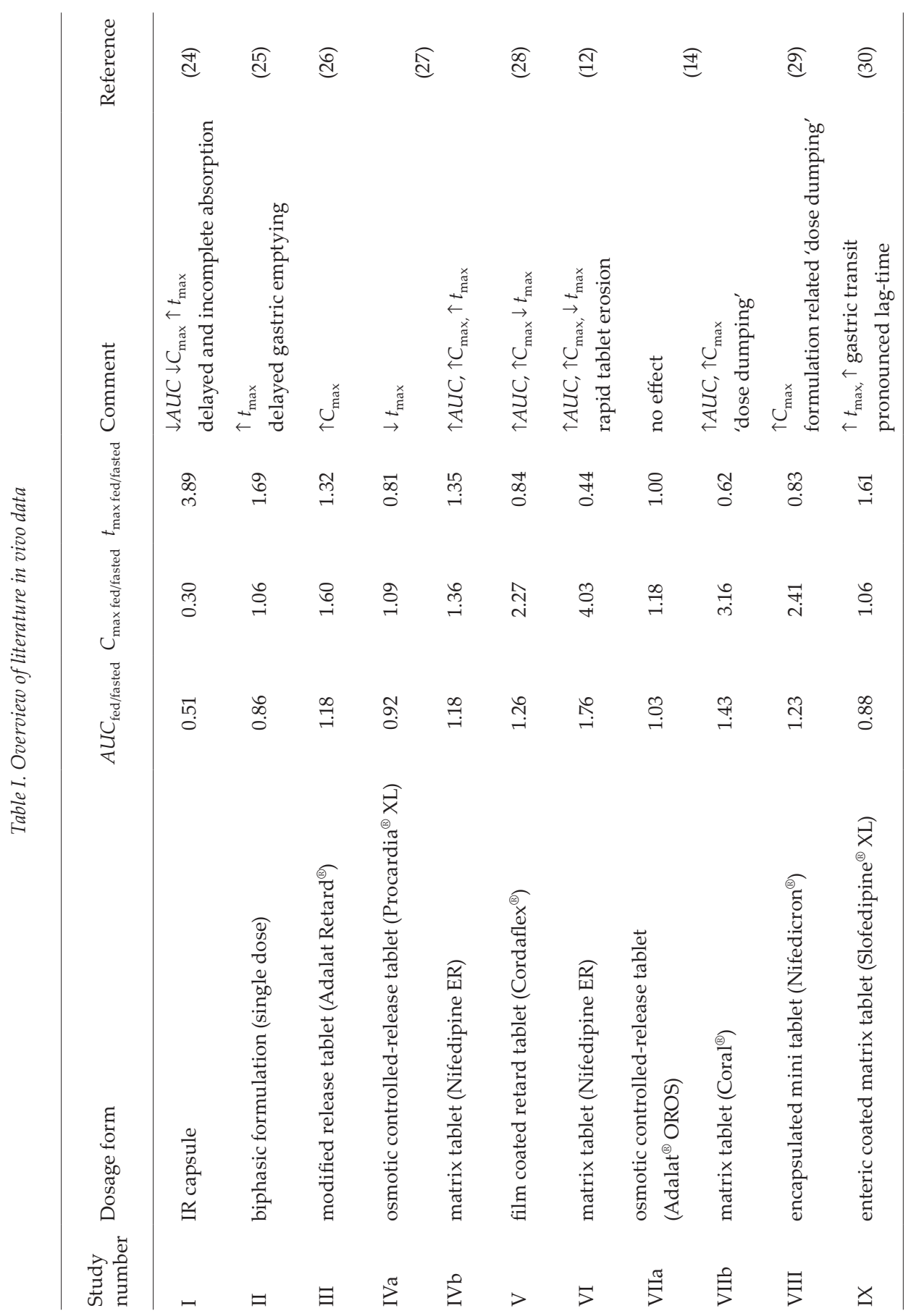



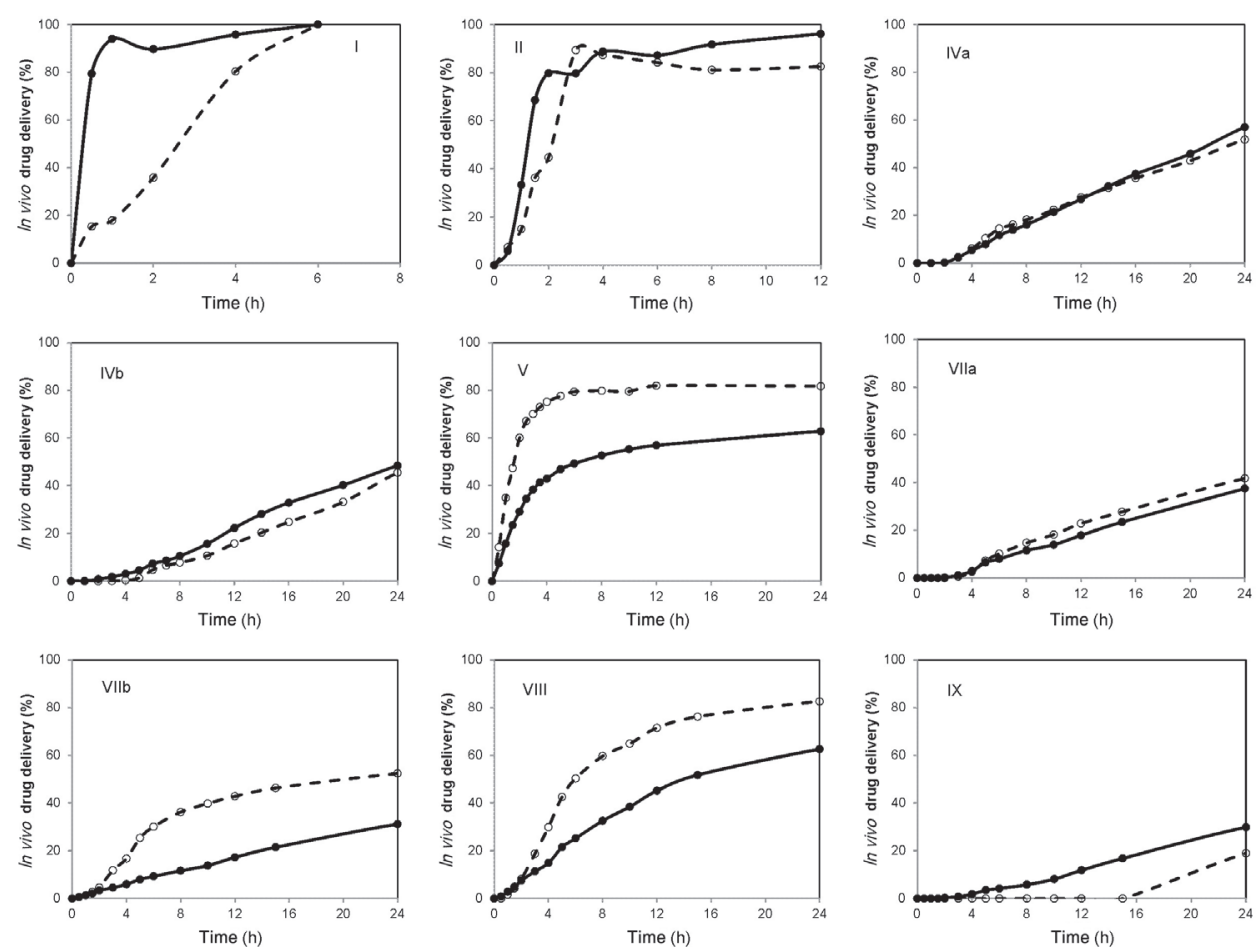

Fig. 2. In vivo drug delivery profiles of various nifedipine products administered in the fasted (full line) or fed state (dotted line) obtained by numerical deconvolution with oral reference. See Table I for key to legend.

drug delivery profiles. In vivo dissolution profiles calculated by numerical deconvolution based on the p.o. reference are given in Fig. 2.

Deconvolution-based methods are particularly helpful for exploratory data analysis, since they provide the cumulative amount absorbed in vivo, which can be used to identify appropriate models for the IVIVC relationship (5). Despite substantial differences in the dosage form design, meal composition and demographic characteristics of the study populations, in vivo input kinetics obtained in the fasted state were relatively consistent: after a relatively short lag time ( 0.5 to 3 hours), nifedipine input followed near zero-order kinetics up to 24 hours, except in the case of IR capsule, biphasic release tablet and film coated retard tablet (studies I, II and V). On the other hand, deviations among the in vivo delivery profiles obtained from the results of the fed state studies were considerable. When drug products which tended to show somewhat faster in vivo dissolution in the fasted state were co-administered with food, they exhibited an increased drug release rate described as »dose dumping " (studies V, VIIb and VIII). In contrast, drug release from the enteric coated matrix tablet was further delayed in the fed state (study IX).

Nifedipine is a highly lipophilic drug, which exhibits a relatively high first pass metabolism. It is expected that the bioavailability of a lipophilic drug characterized by exten- 
sive presystemic metabolism could be enhanced after a meal by a combination of increased solubility and reduced first pass effect $(12,13)$. Using the Biopharmaceutics Drug Disposition Classification System (BDDCS), Custodio et al. (31) predicted an increase in bioavailability of a highly metabolized drug such as nifedipine due to transporter inhibition resulting in reduced extent of drug metabolism.

Abrahamsson et al. (12) found that increased absorption rate, described with the fourfold increase of $C_{\max }$ values in the case of nifedipine ER matrix tablets (Table I, study VI), could be mainly attributed to food affecting the drug release mechanism, i.e., tablet erosion, because close correspondence between tablet erosion and absorption profiles was verified. The authors also found that potential factors which may have induced more rapid erosion included vigorous postprandial gastrointestinal motility and the physicochemical effects of food components and gastric secretions. In further investigations, hydrodynamic mechanical stress was considered to be the main factor responsible for postprandial effects on tablet erosion (10). Schug et al. $(14,29,30)$ reported formulationspecific food effects for different nifedipine modified release preparations. In vivo drug delivery profiles obtained by numerical deconvolution using p.o. reference demonstrate notable differences in fed vs. fasted state (Fig. 2, study VIIb, VIII and IX). The withinproduct comparison of drug bioavailability after fed vs. fasted state administration did not indicate any food effect in the case of the osmotic controlled release system (Adalat ${ }^{\circledR}$ OROS), described by almost superimposable in vivo drug delivery profiles in fasted and fed states (Fig. 2, study VIIa). In contrast, pronounced differences in terms of $A U C$ and $C_{\max }$ values were determined under the fed compared to fasting conditions in the case of matrix tablet formulations (Fig. 2, study VIIb). Food co-administration with the »erosive tablet« caused much faster drug release, described as a »dose dumping « phenomenon (14). Similar results were obtained for the nifedipine extended release formulation containing encapsulated HPMC-based mini tablets, where »a significant loss of modified-release characteristics « was detected under fed conditions (29). In a separate study, it was found that food caused a pronounced delay in nifedipine absorption from Slofedipine ${ }^{\circledR} \mathrm{XL}$, an enteric coated modified release tablet with erosive polymer matrix (30). Since the formulation properties prevent dissolution of the tablet in acidic medium of the stomach, the pronounced lag-time in the presence of food can plausibly be explained by delayed gastric emptying time. Armstrong et al. (26) emphasized the importance of considering the effects of different types of meal before concluding how food affects the pattern of dissolution and absorption of certain drug formulations. Meal composition could also influence the rate of dissolution of some formulations either directly, through precipitation or film formation on the tablet surface $(1,11)$, or indirectly, by affecting secretion of digestive juices, which is more pronounced for modified release formulations $(1,26)$. Current investigations of the bioperformance of nifedipine products are based on integration of silico modeling and in vitro dissolution methodology, using in vivo data previously reported in the literature, as listed in Table I. Published studies revealed that physiologically based pharmacokinetic models developed for nifedipine and coupled with in vitro dissolution data could be successfully used for predicting the in vivo performance of investigated products $(13,17)$.

\section{In vitro dissolution}

Nifedipine dissolution profiles from commercially available osmotic controlled-release tablets and hydrophilic matrix tablets are given in Fig. 3a-b. 
a)

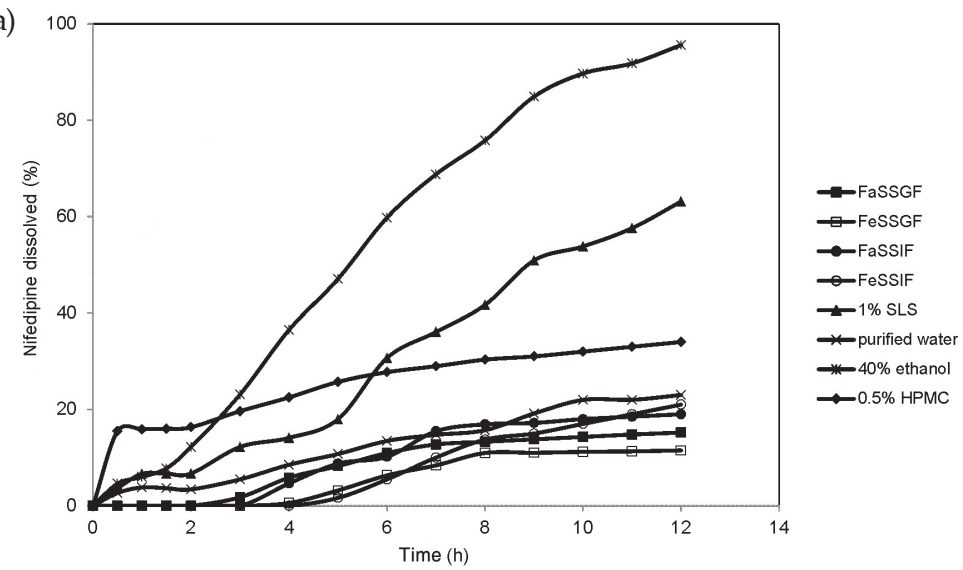

b)

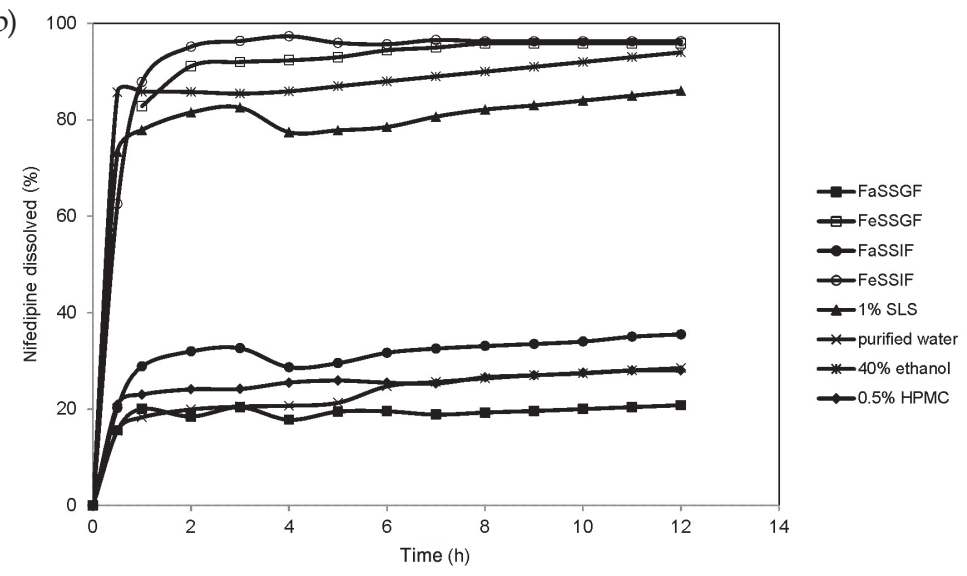

Fig. 3. In vitro dissolution profiles from: a) osmotic controlled-release tablets and b) hydrophilic matrix tablets obtained in various experimental media.

Although the osmotic release drug delivery system has been designed to be robust against the influence of environmental conditions, notable differences in nifedipine dissolution kinetics were observed in various dissolution media, as shown in Fig. 3a. Nifedipine dissolution in aqueous media was slow and incomplete, leading to a total of $23 \%$ dissolved after 12 hours. Such results are in accord with nifedipine solubility in water $(0.01 \mathrm{~g}$ $\left.\mathrm{L}^{-1}\right)$ as reported in the literature $(12,13)$. Minor differences obtained in the fed vs. fasted state simulating media were in accord with the lack of food effect reported in the in vivo studies $(14,27)$. Addition of sodium lauryl sulfate into dissolution media resulted in faster nifedipine release, leading to $63 \%$ drug dissolved in 12 hours. Nifedipine dissolution was also enhanced in viscous media, where an initial burst effect was observed, leading to the deviation of zero order drug release pattern and $34 \%$ drug released after 12 hours. It may be assumed that the reduced hydrodynamic shear rate in viscous media contributed to the 
observed phenomena. Nifedipine release in $40 \%$ ethanol followed zero order kinetics and was substantially faster compared to other media employed, leading to $95 \%$ drug dissolved after 12 hours. The results obtained indicate that nifedipine solubility is not the sole factor affecting its release from the osmotic controlled-release tablets and that other factors such as altered membrane permeability in the presence of ethanol may be contributive.

Nifedipine release from the investigated hydrophilic matrix tablets was highly susceptible to the influence of dissolution media employed. Relevant dissolution profiles are given in Fig. 3b. Drug dissolution was rapid, leading to the maximum amount dissolved after two hours of investigation. However, depending on the dissolution media used, the maximum amount of drug dissolved exhibited a four-fold difference. Total percent of nifedipine dissolved in water and viscous media was slow and incomplete leading to $28 \%$, which is in accord with nifedipine $\mathrm{pH}$ independent aqueous solubility, reported as $0.01 \mathrm{~g}$ $\mathrm{L}^{-1}(12,13)$. Similar results were obtained in the fasted state simulated media, with slightly higher percent dissolved in FaSSIF compared to FaSSGF, i.e., 35 \% compared to $20 \%$, respectively. Nifedipine release in the fed state simulating media was rapid and complete, and the dissolution profiles obtained were almost superimposable regardless of the difference in $\mathrm{pH}$, surface tension and buffer capacity between FeSSGF and FeSSIF, as summarized by Klein (32). Such data are in accord with nifedipine solubility in physiologically based media showing a significant increase of solubility in fed state simulated media compared to fasted conditions (13). Nifedipine dissolution in media containing $1 \%$ SLS was also very fast, leading to $73 \%$ of drug released in 30 minutes, related to the marked increase of nifedipine solubility in the presence of surfactant. Nifedipine dissolution in $40 \%$ ethanol followed the same pattern, leading to rapid and complete drug dissolution in less than 30 minutes. The results obtained indicate that the investigated nifedipine hydrophilic matrix tablets are highly susceptible to the effect of surfactants and ethanol present in the dissolution media. Dissolution data are in accord with the effect on nifedipine solubility of both natural and synthetic surfactants, as well as ethanol. Such dosage form performance is indicative of the potential for dose dumping due to the food effect and/or concomitant alcohol consumption. The results obtained in the physiologically based media simulating fed $v$ fasted state were compliant with the data observed in vivo, where a positive food effect was reported for certain nifedipine hydrophilic matrix formulations $(14,16)$.

\section{Gastrointestinal simulation}

Considering that regulatory approaches to the assessment of bioequivalence are constantly updated, proposing that equivalence may be established by comparing the in vivo drug delivery profiles with application of an equivalence-by-design concept, the importance of reliable iDDP is emphasized, particularly in the evaluation of increasingly complex modified release products. In vitro dissolution data can serve as markers for targeting the desired drug delivery profile in vivo. In this context, the usefulness of gastrointestinal simulation for the prediction of in vivo drug dissolution and absorption was evaluated using the previously developed and validated GastroPlus ${ }^{\mathrm{TM}}$ model (21). In vitro dissolution data of investigated dosage forms obtained in the present study were used as input values for GIS. GastroPlus ${ }^{\mathrm{TM}}$ model was used to simulate nifedipine plasma concentration profiles of the osmotic controlled-release and hydrophilic matrix tablets in order to evaluate the biorelevance of investigated dissolution media. Predicted $c-t$ profiles obtained with GIS 

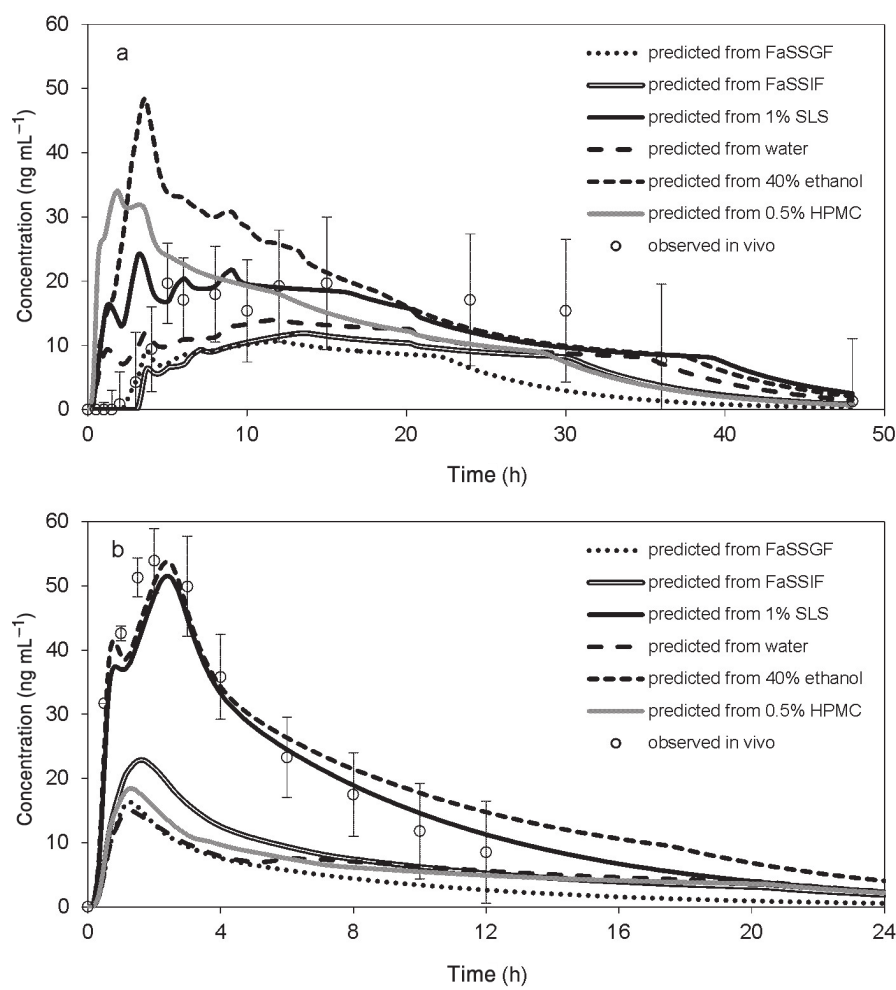

Fig. 4. Plasma concentration profiles of: a) osmotic controlled-release tablets and b) hydrophilic matrix tablets predicted using the GastroPlus ${ }^{\mathrm{TM}}$ model under fasting condition.

under fasting conditions for osmotic controlled-release and hydrophilic matrix tablets are shown in Figs. $4 a$ and $4 b$, respectively.

The best fit of the predicted plasma concentration profile and those observed in vivo for osmotic controlled-release tablets (14) and hydrophilic matrix tablets (33) was obtained with the dissolution profile in $1 \%$ SLS used as input function in GIS. Predictability of the generated absorption model was measured by the percent prediction error (\% PE) between the predicted and in vivo observed data. The predicted pharmacokinetic parameters $C_{\max }$ and $A U C_{0 \text {-inf }}$ for osmotic controlled-release tablets were very close to the values obtained in vivo, in both fasted and fed states, described by the PE values less than $10 \%$, indicating good predictability, as shown in Table II.

Differences in $C_{\max }$ values were caused by the occurrence of double peaks, which may be related to physiological events and first pass metabolism. Simulated $c-t$ profiles for hydrophilic matrix tablets described with pharmacokinetic parameters close to those observed in vivo (33) suggest generalization ability of the GIS model used. The results obtained indicate the potential of using the developed physiologically based model for simulation of iDDP and in vivo product behavior based on discriminative in vitro dissolution data. 
M. Ilić et al.: Deciphering nifedipine in vivo delivery from modified release dosage forms: Identification of food effect, Acta Pharm. 65 (2015) 427-441.

Table II. In vivo observed and in silico (GIS) predicted pharmacokinetic parameters using $1 \%$ SLS as dissolution media

\begin{tabular}{lcccccc}
\hline $\begin{array}{l}\text { Pharmacokinetic } \\
\text { parameter }\end{array}$ & \multicolumn{3}{c}{$C_{\max }\left(\mathrm{ng} \mathrm{mL}^{-1}\right)$} & \multicolumn{3}{c}{$A U C_{0 \text {-inf }}\left(\mathrm{ng} \mathrm{h} \mathrm{\textrm {mL } ^ { - 1 } )}\right.$} \\
\hline Osmotic tablets & Observed & Predicted & $\%$ PE & Observed & Predicted & $\%$ PE \\
Fasting state & 19.66 & 24.31 & 23.65 & 598.33 & 617.35 & 3.18 \\
Fed state & 24.79 & 25.53 & 2.98 & 609.99 & 647.13 & 6.09 \\
Matrix tablets & & & & & & \\
Fasting state & 53.90 & 51.53 & 4.40 & 372.35 & 395.37 & 6.18 \\
\hline
\end{tabular}

\section{CONCLUSIONS}

The in vivo drug absorption/dissolution profile represents a basis for bioperformance dissolution test development and establishment of an in vitro/in vivo correlation model. Therefore, reliable estimation of in vivo drug delivery is an important factor in the biopharmaceutical characterization of drug products. Comparative evaluation of the results obtained by applying conventional pharmacokinetic methods and in silico gastrointestinal simulation revealed that, although the general pattern of drug delivery was similar, its extent deciphered by different techniques varied. Having in mind the effect of presystemic metabolism on nifedipine bioavailability, the drug delivery profile estimated by numerical deconvolution with the immediate release capsule administration used as a reference appears to be advantageous. The data presented in this study demonstrate that the in silico model developed can be successfully used to complement relevant in vitro studies in the simulation of nifedipine in vivo delivery from the investigated dosage form.

In vivo study reports indicate a physicochemically based, formulation specific food effect of nifedipine modified release tablets. A number of potential interaction mechanisms have been suggested.

Nifedipine in vitro release from the investigated dosage forms was susceptible to the influence of dissolution media employed, corresponding to the distinctly different release mechanisms. Interestingly, in vitro dissolution profiles obtained in $1 \%$ SLS used as input in the in silico gastrointestinal simulation model provided discriminatory power necessary for good predictability of the in vivo dosage form performance for both osmotic release and hydrophilic matrix tablets. The results obtained indicate that, in certain cases, bioperformance dissolution can be achieved using relatively simple dissolution media that provide the prevailing factor governing drug release without the need to closely mimic in vivo conditions with respect to medium $\mathrm{pH}$ and composition.

Acknowledgements. - This work was done under Project TR-34007 supported by the Ministry of Education, Science and Technological Development, Republic of Serbia. 


\section{REFERENCES}

1. D. Fleisher, C. Li, Y. Zhou, L. H. Pao and A. Karim, Drug, meal and formulation interactions influencing drug absorption after oral administration clinical implications, Clin. Pharmacokinet. 36 (1999) 233-254; DOI: 10.2165/00003088-199936030-00004.

2. K. A. Lentz, Current methods for predicting human food effect, The AAPS Journal 10 (2008) 282288; DOI: 10.1208/s12248-008-9025-8.

3. FDA/CDER (2002), Guidance for Industry: Food-effect Bioavailability and Fed Bioequivalence Studies.

4. EMA/CPMP (2010), Guideline on the Investigation of Bioequivalence, CPMP/EWP/QWP/1401/98 Rev. $1 /$ Corr $^{* *}$.

5. EMA/CHMP (2014), Guideline on the Pharmacokinetic and Clinical Evaluation of Modified Release Dosage Forms, EMA/CPMP/EWP/280/96 Corr1.

6. W. Jiang, S. Kim, X. Zhang, R. A. Lionberger, B. M. Davit, D. P. Conner and L. X. Yu, The role of predictive biopharmaceutical modeling and simulation in drug development and regulatory evaluation, Int. J. Pharm. 418 (2011) 151-160; DOI: 10.1016/j.ijpharm.2011.07.024.

7. E. S. Kostewicz, L. Aarons, M. Bergstrand, M. B. Bolger, A. Galetin, O. Hatley, M. Jamei, R. Lloyd, X. Pepin, A. Rostami-Hodjegan, E. Sjögren, C. Tannergren, D. B. Turner, C. Wagner, W. Weitschies and J. Dressman, PBPK models for the prediction of in vivo performance of oral dosage forms, Eur. J. Pharm. Sci. 57 (2014) 300-321; DOI: 10.1016/j.ejps.2013.09.008.

8. E. S. Kostewicz, B. Abrahamsson, M. Brewster, J. Brouwers, J. Butler, S. Carlert, P. A. Dickinson, J. Dressman, R. Holm, S. Klein, J. Mann, M. McAllister, M. Minekus, U. Muenster, A. Müllertz, M. Verwei, M. Vertzoni, W. Weitschies and P. Augustijns, In vitro models for the prediction of in vivo performance of oral dosage forms, Eur. J. Pharm. Sci. 57 (2014) 342-366; DOI: 10.1016/j. ejps.2013.08.024.

9. EMA, QWP (2012), Guideline on Quality of Oral Modified Release Products, Draft, EMA/ 492713/2012.

10. B. Abrahamsson, K. Roos and J. Sjogren, Investigation of prandial effects on hydrophilic matrix tablets, Drug Dev. Ind. Pharm. 25 (1999) 765-771; DOI: 10.1081/DDC-100102236.

11. J. Parojčić, D. Vasiljević, S. Ibrić and Z. Đurić, Tablet disintegration and drug dissolution in viscous media: Paracetamol IR tablets, Int. J. Pharm. 355 (2008) 93-99; DOI: 10.1016/j.ijpharm.2007.11.058.

12. B. Abrahamsson, M. Alpsten, B. Bake, U. E. Jonsson, M. Eriksson-Lepkowska and A. Larsson, Drug absorption from nifedipine hydrophilic matrix extended-release (ER) tablet - comparison with an osmotic pump tablet and effect of food, J. Control. Rel. 52 (1998) 301-310; DOI: 10.1016/ S0168-3659(97)00267-8.

13. C. Wagner, K. Thelen, S. Willmann, A. Selen and J. B. Dressman, Utilizing in vitro and PBPK tools to link ADME characteristics to plasma profiles: Case example nifedipine immediate release formulation, J. Pharm. Sci. 102 (2013) 3205-3219; DOI: 10.1002/jps.23611.

14. B. S. Schug, E. Brendel, D. Wolf, M. Wonnemann, M. Wargenau and H. H. Blume, Formulationdependent food effects demonstrated for nifedipine modified-release preparations marketed in the European Union, Eur. J. Pharm. Sci. 15 (2002) 279-285; DOI: 10.1016/S0928-0987(02)00008-8.

15. M. Wonnemann, B. Schug, K. Schmücker, E. Brendel, P. A. van Zwieten and H. Blume, Significant food interactions observed with a nifedipine modified-release formulation marketed in the European Union, Int. J. Clin. Pharmacol. Ther. 44 (2006) 38-48; DOI: 10.5414/CPP44038.

16. M. Wonnemann, B. S. Schug, M. Anschutz, E. Brendel, G. De Nucci and H. H. Blume, Comparison of two marketed nifedipine modified-release formulations: An exploratory clinical food interaction study, Clin. Ther. 30 (2008) 48-58; DOI: 10.1016/j.clinthera.2008.01.001.

17. N. Patel, S. Polak, M. Jamei, A. Rostami Hodjegan and D. B. Turner, Quantitative prediction of formulation-specific food effects and their population variability from in vitro data with the phys- 
iologically-based ADAM model: A case study using the BCS/BDDCS Class II drug nifedipine, Eur. J. Pharm. Sci. 57 (2014) 240-249; DOI: 10.1016/j.ejps.2013.09.006.

18. T. J. Rashid, U. Martin, H. Clarke, D. G. Waller, A. G. Renwick and C. F. George, Factors affecting the absolute bioavailability of nifedipine, Br. J. Clin. Pharmacol. 40 (1995) 51-58; DOI: 10.1111/j.13652125.1995.tb04534.x.

19. J. C. Loo and S. Riegelman, New method for calculating the intrinsic absorption rate of drugs, J. Pharm. Sci. 57 (1968) 918-928; DOI: 10.1002/jps.2600570602.

20. B. Agoram, W. S. Woltosz and M. B. Bolger, Predicting the impact of physiological and biochemical processes on oral drug bioavailability, Adv. Drug Deliv. Rev. 50 (2001) S41-S67; DOI: 10.1016/ S0169-409X(01)00179-X.

21. M. Ilić, J. Đuriš, I. Kovačević, S. Ibrić and J. Parojčić, In vitro - in silico - in vivo drug absorption model development based on mechanistic gastrointestinal simulation and artificial neural networks: Nifedipine osmotic release tablets case study, Eur. J. Pharm. Sci. 62 (2014) 212-218; DOI: 10.1016/j.ejps.2014.05.030.

22. E. Jantratid, N. Janssen, C. Reppas and J. B. Dressman, Dissolution media simulating conditions in the proximal human gastrointestinal tract: an update, Pharm. Res. 25 (2008) 1663-1676; DOI: 10.1007/s11095-008-9569-4.

23. Summary of Product Characteristics: Adalat modified release tablets, Bayer plc, revision date April 12, 2012 (www.medicines.org.uk).

24. K. Hirasawa, W. F. Shen, D. T. Kelly, G. Roubin, K. Tateda and J. Shibata, Effect of food ingestion on nifedipine absorption and haemodynamic response, Eur. J. Clin. Pharmacol. 28 (1985) 105-107; DOI: 10.1007/BF00635716.

25. V. Challenor, D. G. Waller, B. S. Gruchy, A. G. Renwick, C. F. George, E. T. McMurdo and J. McEwen, The effect of food and posture on the pharmacokinetics of a biphasic release preparation of nifedipine, Br. J. Clin. Pharmacol. 22 (1986) 565-570; DOI: 10.1111/j.1365-2125.1986.tb02936.x.

26. J. Armstrong, V. F. Challenor, B. S. Macklin, A. G. Renwick and D. G. Waller, The influence of two types of meal on the pharmacokinetics of a modified-release formulation of nifedipine (Adalat Retard), Eur. J. Clin. Pharmacol. 53 (1997) 141-143; DOI: 10.1007/s002280050352.

27. FDA/CDER (1998), Bioequivalence Review: Application Number 75-108.

28. K. B. Nemes, V. Horvath, G. Grezal, G. Horvai, A. Hrabeczy-Pall, E. Kocsi, S. Drabant, M. Csorgo, G. Rencez and I. Klebovich, Food interaction pharmacokinetic study of Cordaflex $20 \mathrm{mg}$ retard film tablet in healthy volunteers, Int. J. Clin. Pharmacol. Ther. 36 (1998) 263-269.

29. B. S. Schug, E. Brendel, M. Wonnemann, D. Wolf, M. Wargenau, A. Dingler and H. H. Blume, Dosage form-related food interaction observed in a marketed once-daily nifedipine formulation after a high-fat American breakfast, Eur. J. Clin. Pharmacol. 58 (2002) 119-125; DOI: 10.1007/s00228-0020444-7.

30. B. S. Schug, E. Brendel, E. Chantraine, D. Wolf, W. Martin, R. Schall and H. H. Blume, The effect of food on the pharmacokinetics of nifedipine in two slow release formulations: pronounced lagtime after a high fat breakfast, Br. J. Clin. Pharmacol. 53 (2002) 582-588; DOI: 10.1046/j.1365-2125. 2002.01599.x.

31. J. M. Custodio, C. Y. Wu and L. Z. Benet, Predicting drug disposition, absorption/elimination/ transporter interplay and the role of food on drug absorption, Adv. Drug Deliv. Rev. 60 (2008) 717-733; DOI: 10.1016/j.addr.2007.08.043.

32. S. Klein, The use of biorelevant dissolution media to forecast the in vivo performance of a drug, The AAPS Journal 12 (2010) 397-406; DOI: 10.1208/s12248-010-9203-3.

33. C. Piovella, Clinical efficacy and bioavailability of a sustained release nifedipine formulation, Arzneimittelforschung 37 (1987) 832-835. 